Casos Clínicos

Arch. Esp. Urol., 61, 8 (932-936), 2008

\title{
PSEUDOTUMOR FIBROSO PARATESTICULAR
}

Miguel Ángel López Aramburu, Amparo Viguri Díaz', Pablo Peña Pérez², José, Rosa Arias, y Mónica Saiz Camin'.

Servicio de Urología. Servicio de Anatomía Patológica' y Enfermería Urológica². Hospital Santiago Apóstol. Miranda de Ebro. Burgos. España.

Resumen.- OBJETIVOS: Los procesos proliferativos benignos testiculares se conocen con el nombre de pseudotumores, al no ser considerados en la actualidad verdaderas neoplasias. El Pseudotumor Fibroso Paratesticular (PFP) es una entidad sobre las que existe gran confusión en su definición y etiopatogenia. Se trata de un proceso que confunde al clínico al aparecer simulando neoplasias. La presente comunicación puede ayudar a conocer mejor este proceso no tumoral y evitar confusiones diagnósticas.

MÉTODOS/RESULTADOS: Revisamos nuestra serie de PFP (tres casos) sobre esta rara entidad, por lo que dada su rareza puede considerarse significativa. Analizamos su clínica, su comportamiento antes los estudios diagnósticos por imagen, hallazgos operatorios y evolución.

CONCLUSIONES. EI PFP es una rara entidad que puede confundir al clínico al ser diagnosticado como una neopla-

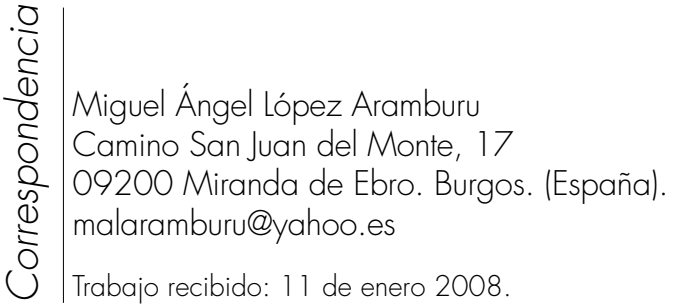


sia, y conllevar tratamientos agresivos (orquiectomía) que de realizar un exacto diagnóstico podrían obviarse. El análisis de una serie de tres casos, con sus aportaciones, y la revisión de la literatura efectuada pueden ayudar en la practica clínica al urólogo a reconocer esta enfermedad.

Palabras clave: Testículo. Pseudotumores. Pseudotumor Fibroso.

Summary.- OBJECTIVE: Benign testicular proliferative processes are known by the name of pseudotumors, because currently they are not considered real neoplasias. The paratesticular fibrous pseudotumor (PFP) is a rare entity, the definition and etiopathogenesis of which is under great confusion. It is a process that misleads the clinical because they simulate neoplasias. This paper aims to help a better knowledge of this non-tumoral process and to avoid diagnostic confusions.

METHODS/RESULTS: We review our series of PFP /three cases), which can be considered significant due to its oddity. We analyze the clinical picture, its behavior, imaging diagnostic tests, operative findings and outcomes.

CONCLUSIONS: PFP is a rare entity which may misleads the clinical because it may be diagnosed as a neoplasia and lead to aggressive treatment (orchiectomy) which in case of a proper diagnosis could be avoided. The analysis of our series of three cases, with their contribution, and a literature review may help the clinical practice of urologists by recognizing this disease.

Keywords: Testicle. Pseudotumors. Fibrous pseudotumor.

\section{INTRODUCCIÓN}

El Pseudotumor Fibroso Paratesticular (PFP) es una entidad patológica sobre la que se ha cernido un gran confusionismo. Actualmente se considera un proceso reactivo inflamatorio no tumoral aunque no existe unanimidad al respecto (1-4).

En primer lugar llamamos la atención sobre las múltiples y diversas denominaciones que en la literatura medica podemos encontrar para el PFP, a saber:

Fibroma, periorquitis pseudofibromatosa, periorquitis reactiva, pseudotumor inflamatorio, pseudotumor paratesticular, periorquitis crónica proliferativa, proliferación difusa y nodular etc..

Es una lesión que confunde al clínico al simular, en no pocas ocasiones, un tumor testicular, y es tratado en tales ocasiones con tratamientos quirúrgicos radicales, que podrían obviarse si se realizase un correcto diagnóstico preoperatorio, que aunque no es fácil puede hacerse si conocemos esta enfermedad. Con este fin presentamos nuestra casuística (5-8).

\section{MATERIAL Y MÉTODOS}

\section{Caso 1}

Paciente de 89 años de edad con antecedente de colocación de prótesis de cadera derecha por coxartrosis realizada hace 7 años. Hace 2 fue sometido en nuestro Servicio a amputación parcial de pene por carcinoma escamoso bien diferenciado queratinizante de tipo verrucoso, presentando desde entonces una excelente evolución con control de la enfermedad.

En el momento actual presenta hidrocele bilateral. La ecografía nos informa que en el lado derecho presenta tabicación y una imagen esférica de 17 mm. de diámetro con centro calcificado y periferia hipoecoica. En el lado derecho se detecta otra imagen similar a la anterior de $8 \mathrm{~mm}$. de diámetro, desplazándose ambas libremente en la cavidad del hidrocele. Asimismo se detecta al nivel de la superficie testicular (albugínea) derecha una zona hipoecoica de límites indefinidos con tenue sombra posterior. Con el diagnóstico de Hidrocele bilateral se procede a intervención descubriéndose dos nódulos intravaginales (uno en cada cavidad testicular) de consistencia pétrea (Figura 1) y una zona indurada y engrosada en la albugínea testicular que se reseca y envía a anatomía patológica. Este nos informa que microscópicamente se estudian varios fragmentos de tejido conectivo en su mayor parte con marcada hialinización salpicado con pequeños vasos capilares y leve infiltrado inflamatorio de linfocitos y ocasionales células plasmáticas. Además se estudian dos formaciones nodulares

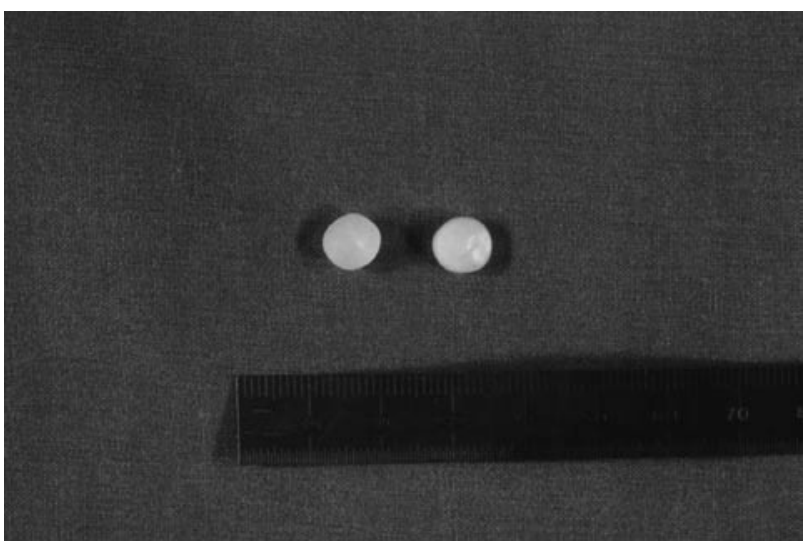

FIGURA 1. Cuerpos libres intraescrotales (caso 1). 
constituidas por un tejido fibroso virtualmente acelular, con marcada hialinización. Aislados vasos capilares y leve infiltrado inflamatorio linfoplasmocitario. En el área central calcificación distrófica: Pseudotumor Fibroso Paratesticular.

\section{Caso 2:}

Paciente de 68 años con antecedentes de cólicos renales, que consulta por molestias testiculares leves e inespecíficas. La exploración no aporta datos de interés excepto discreto engrosamiento de cabeza epidídimo derecho sensible a la palpación. El estudio ecográfico nos informa de la existencia de imágenes redondeadas adosadas a la albugínea testicular y vaginal. Pequeño hidrocele bilateral y quiste en cabeza de epidídimo derecho. Se programa extirpación-biopsia de las lesiones descritas.

Por escrototomía se procede a exploración observando la presencia de pequeñas formaciones nodulares aplanadas que se extienden por la albugínea testicular procediéndose a extirpación de las de mayor tamaño (diámetro aproximado de $5 \mathrm{~mm}$.). El Servicio de Anatomía Patológica nos informa que ha procedido a estudiar microscópicamente varias formaciones nodulares constituidas por fibroblastos entremezclados con abundantes vasos, focos de hemorragia reciente y antigua. En algunas áreas se continúan con un tejido fibromuscular con áreas hialinizadas y moderado infiltrado inflamatorio linfoplasmocitario: Pseudotumor fibroso (Figura 2).

\section{Caso 3:}

Paciente de 64 años de edad con antecedentes de artrodesis de CV por escoliosis grave, que consulta por síndrome de dificultad miccional. Además refiere la presencia de un nódulo móvil en hemiescroto izquierdo, que le produce ligeras molestias inespecíficas leves, pero que le preocupan enormemente.

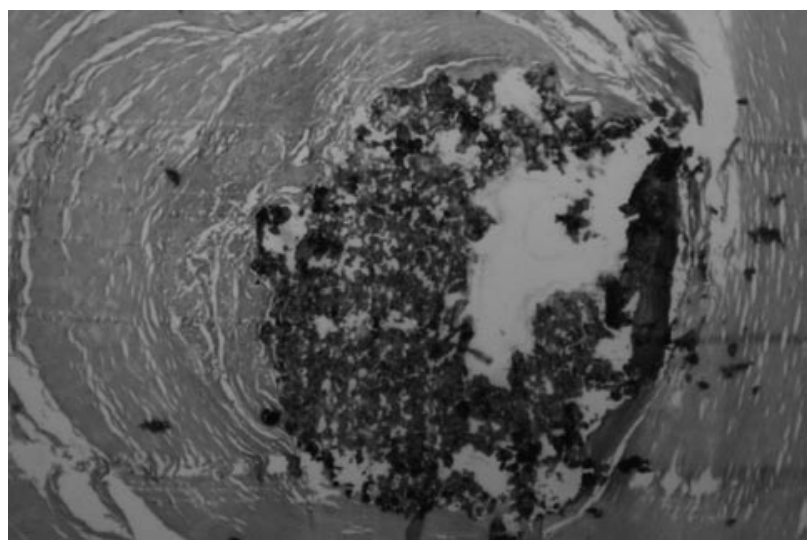

FIGURA 2. Aspecto microscópico del pseudotumor fibroso: Tejido conectivo hialinizado y calcificación distrófica. H/E x 200.
La exploración escrotal pone en evidencia un nódulo duro de aproximadamente un centímetro de diámetro, de consistencia pétrea y totalmente móvil.

Ecografía escrotal: Testículos normales. Cuerpo libre en el lado izquierdo con ecoestructura de tejido blando, salvo por un núcleo central ecogénico con tenue sombra compatible con foco calcificado (Figura 3).

Bajo anestesia local y con el diagnóstico de pseudotumor fibroso paratesticular es sometido a cirugía practicándose exéresis de formación nodular (Figuras 4 y 5 ) que anatomía patológica informa como lesión nodular escasamente celular constituida por colágeno hialinizado con calcificación distrófica en su zona central (Pseudotumor fibroso paratesticular).

\section{RESULTADOS}

Presentamos tres casos de pseudotumor fibroso paratesticular. En sólo uno se realizó diagnóstico preoperatorio correcto (caso 3). Los dos restantes fueron operados con los diagnósticos de hidrocele y nódulos testiculares a estudio (casos 1 y 2). En ninguno se barajó como posibilidad diagnóstica la presencia de tumor maligno. A ningún paciente se le indicó cirugía agresiva de exéresis. Todos los pacientes presentaron molestias testiculares leves.

Los hallazgos ecográficos fueron en dos casos lo suficientemente claras para realizar el diagnóstico (casos 1 y 3) ya que se nos informaba de la presencia de cuerpos libres intravaginales, lo que constituye un dato muy orientativo, pero solo en el caso 3 reconocimos la entidad, basándonos indudablemente en la experiencia acumulada.

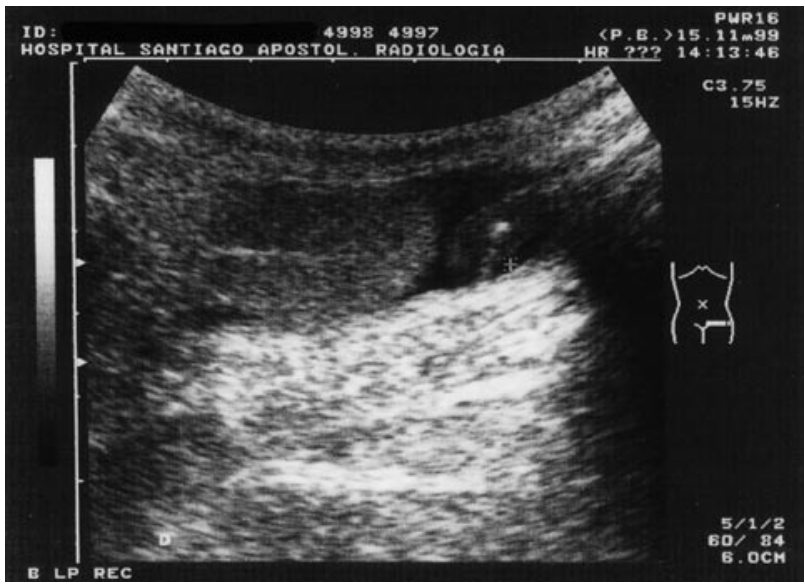

FIGURA 3. Imagen ecográfica de cuerpo libre-nódulo intraescrotal adyacente al testículo. En su parte central núcleo ecogénico: foco de calcificación. 


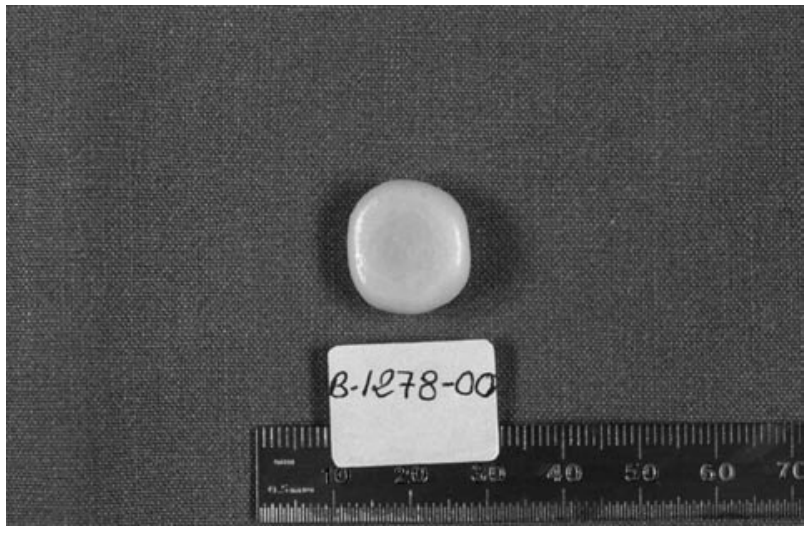

FIGURA 4. Cuerpo libre intraescrotal (caso 3).

Ningún paciente presentó complicación alguna y los tres presentaron una excelente evolución.

\section{DISCUSIÓN}

El PFP es un proceso proliferativo benigno de origen incierto, probablemente inflamatorio reactivo no tumoral, aunque no existe unanimidad en este aspecto y algunos autores lo consideran un proceso neoplásico. No obstante, esta teoría parece perder peso últimamente a favor del origen reactivo-inflamatorio $(1,2,4,6,9)$.

No obstante su benignidad, en no pocos casos simula clínicamente un tumor testicular, y no han sido pocos los pacientes que han sido sometidos a cirugías radicales de exéresis, al realizarse un diagnóstico de neoplasia testicular cuando la entidad era el $\operatorname{PFP}(3,5,7)$.

Es un proceso infrecuente pero no excepcional. Afecta a los varones en todas las edades pero más frecuentemente entre $3^{\underline{a}}$ y $6^{\underline{a}}$ década de la vida.

Afecta a túnica testiculares y más raramente a epidídimo y cordón espermático. Se presenta como nódulo o nódulos firmes (duros) irregularmente repartidos sobre túnica albugínea. Pueden acompañarse de la presencia de cuerpos libres en la cavidad vaginal. Son asintomáticos o producen leves molestias testiculares inespecíficas. Se asocian en la mitad de los casos con hidrocele. Puede haber o no historia previa de traumas, inflamación, infección etc. $(2,4,6)$.

Ecografricamente se detectan como engrosamientos focales (placas) de ecogenicidad heterogénea y sólida poco orientativa, que miden entre medio y 8 centímetros. En otros casos puede presentarse como nódulos con morfología polilobulada. El parénquima testicular no presenta alteraciones y al TAC suele manifestarse

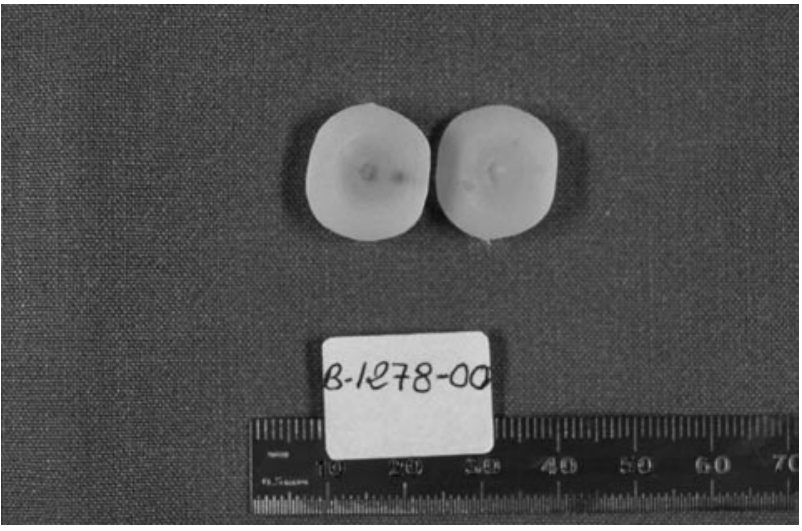

FIGURA 5. Corte del cuerpo libre del caso 3. Se aprecia su zona central calcificada.

como lesión hipodensa. En la RNM da señal de baja intensidad en T1 y T2 (signo de fibrosis), aunque existe poca experiencia al respecto. Marcadores negativos.

Histológicamente presenta tejido de proliferación fibroblástico en un estroma con extensa producción de colágeno, capilares pequeños y células de extirpe inflamatoria crónica (células plasmáticas, linfocitos, histiocitos y eosinófilos) con diversos grados de maduración según el tiempo de evolución. Desde tejido de granulación organizado a tejido completamente hialinizado. En ocasiones se han observado calcificaciones distróficas u osificación focal (1,3 y4).

\section{CONCLUSIONES}

El pseudotumor fibroso paratesticular es una entidad clínica perfectamente individualizada y sobre la que se ha cernido un gran confusionismo. Es un proceso proliferiferativo benigno inflamatorio reactivo y no tumoral.

Su forma de presentación y sus características histológicas varían dependiendo del estadio evolutivo en el que se produzca el diagnóstico.

Clínicamente pude presentarse desde nódulos o engrosamientos de la albugínea hasta masas compactas.

Histológicamente se constata la presencia de tejido de proliferación fibroblástico en un estroma con gran producción de colágeno. Cualquier tipo de célula de extirpe inflamatoria puede estar presente, y en diversos grados de maduración.

Cuando se presenta en estadios evolutivos avanzados como masas compactas el diagnostico diferencial con las neoplasias testiculares es muy difícil, y en estos casos la orquiectomia es difícilmente evitable, ya que no disponemos de pruebas diagnosticas diferenciales, y su 
comportamiento frente a los estudios de imagen son sugestivas de proceso neoplásico.

En los estadios evolutivos más precoces el diagnostico es posible. Su asociación a hidrocele y la frecuente asociación a cuerpos libres intraescrotales, así como la presencia de un parénquima testicular indemne en la ecografía pueden ayudar al diagnostico.

Los tratamientos deben ser siempre conservadores dada su benignidad.

\section{BIBLIOGRAFÍA y LECTURAS RECOMENDADAS (*lectura de interés $y$ ** lectura fundamental)}

**1. RUBENSTEIN, R.A.; DOGRA, V.S.; SEFTEL, A.D. y cols.: "Benign intrascrotal lesions". J. Urol., 171: 1765, 2004.

*2. SANZ JACA, J.P.; ALDAVE VILLANUEVA, J.; ARRINDA YERREGUI, J.M. y cols.: "Pseudotumor fibroso de la túnica vaginal testicular". Arch. Esp. Urol., 43: 786, 1990.

3. RODRÍGUEZ-FERNANDEZ, A.M.; SEVILLA-CHICA, F.I.: "Pseudotumor fibroso paratesticular". Patología, 25: 53, 1992.

4. SÁENZ DE SANTAMARÍA MORALES, F.J.; SÁNCHEZ CHAPADO, M.; PARRA PÉREZ, M. y cols.: "Pseudotumor fibroso de cordón espermático. Estudio morfológico e inmunohistoquímico de un caso y revisión de la literatura”. Arch. Esp. Urol., 41: 233, 1988.

5. RAMOS MARTÍN, J.A.; GARCÍA GONZÁLEZ, A.; JIMÉNEZ DÍAZ, J.: "Pseudotumor fibroso de testículo: Descripción de un caso". Arch. Esp. Urol., 44: 81, 1991.

**6. PELLICER VILALTA, C.; COSME JIMÉNEZ, M.; CASALOTS SERRAMIA, J.: "Neoplasias de las túnicas del testículo. A propósito de cinco casos (tres tumores mesoteliales y dos pseudotumores fibrosos)". Arch. Esp. Urol, 49: 12, 1996.

7. GARCÍA CARRIAZO, M.; BUSTOS GÓMEZ, C.; DOMÍNGUEZ FRANJO, E. y cols.: "Pseudotumor fibroso de la túnica vaginal del testículo. Presentación de un nuevo caso y revisión de la literatura". Actas Urol. Esp., 68, 1999.

8. GARCÍA TORRELLES, M.; BELTRÁN ARMADA, J.R.; SANTOLAYA GARCÍA, I. y cols.: "Tumor fibroso solitario de la túnica vaginal”. Arch. Esp. Urol., 59: 186, 2006.

9. ORTIZ RODRÍGUEZ-PARETS, J.; SILVA ABUIN, J.; ABAD HERNÁNDEZ, M. y cols.: "Periorquitis fibromatosa (pseudotumor fibroso testicular)". Arch. Esp. Urol., 55: 847, 2002. 